\title{
Applications of ToF-SIMS in a Research and Development Laboratory
}

\author{
V.S. Smentkowski and S.G. Ostrowski \\ General Electric Global Research Center, Niskayuna, NY 12309
}

Time of flight secondary ion mass spectrometry (ToF-SIMS) has a number of desirable characteristics for surface analysis including: high surface specificity (analysis of the top 0.3 to 1.0 $\mathrm{nm}$ of samples), high sensitivity (ppb for most elements), high mass resolution $(\mathrm{M} / \mathrm{M}>10,000$ ), high $(<100 \mathrm{~nm})$ lateral resolution, and the ability to detect all elements (including hydrogen) plus intact, high mass species during a single measurement [1]. Commercial ToF-SIMS instruments can operate in a spectroscopy mode, an imaging mode, or a depth profile mode. At each pixel in an image (or each depth in a depth profile) an entire mass spectrum is saved, ToF-SIMS data sets are very large and contain a wealth of information regarding the sample being analyzed.

In this presentation, we show the benefits of ToF-SIMS analysis via examples. Figure 1 (left panel) shows an optical image of an electronic assembly. The analytical team needed to determine if copper was present on the sample. Prior to ToF-SIMS, the sample was analyzed using SEM/EDX, XRF, ICP-MS, and Auger electron spectroscopy; $\mathrm{Cu}$ was not detected. The ToF-SIMS spectrum (middle panel Figure 1) clearly showed a peak at 62.93 amu corresponding to $\mathrm{Cu}$. $\mathrm{C}_{5} \mathrm{H}_{3}$ is detected at $63.02 \mathrm{amu}$; high mass resolution mass spectrometry was needed in order to positively detect $\mathrm{Cu}$. An elemental ion image of $\mathrm{Cu}$ is shown in the middle panel. The far right panel shows that $\mathrm{Cu}$ is localized on the Au pad. The Cu concentration for this example was less than $900 \mathrm{ppb}$.

Figure 2 shows data collected on a polymer sample that contained $1 \%$ of a high mass additive (BnPOSS, formula $=\mathrm{M}=\mathrm{Si}_{8} \mathrm{O}_{12} \mathrm{C}_{56} \mathrm{H}_{56}$ ). The spectrum clearly reveals the molecular peak at 1,145.2 amu as well as characteristic fragment ions. BnPOSS dimer is also detected at 2,290.4 amu (not shown here). ToF-SIMS images of intact BnPOSS (as well as other higher yield molecular fragments) are shown in Figure 2. This example shows the ability to analyze high mass species using ToF-SIMS.

Figure 3 shows a depth profile of trace quantities $\left(0.6\right.$ to $6 \times 10^{21}$ atoms/cc) of $\mathrm{P}$ in a 3.2 micron thick multilayer assembly (left) and an optical profilometer image (right) of the resulting crater. The 200 micron x 300 micron sputter crater was generated using $3 \mathrm{kV} \mathrm{Cs}^{+}$. It is of interest to note the sharpness of the crater wall in the optical profilometer image and the sharpness of the layer interfaces (in the ToF-SIMS depth profile).

In this presentation, we also demonstrate that Multivariate Statistical Analysis tools are useful for analyzing the entire measured data sets $[2,3]$.

References

[1] J.C. Vickerman and D. Briggs, Eds. ToF-SIMS Surface Analysis by Mass Spectrometry, Surface Spectra/IMPublications, London, 2001.

[2] V.S. Smentkowski et al., Anal. Chem. 77 (2005) 1530

[3] P.G. Kotula et al., Microsc. Microanal. 9 (2003) 1. 
$\dagger$ We would like to thank Rosalyn Klinger (GE-GRC) for performing the optical profilometer measurements of the depth profile crater shown here.
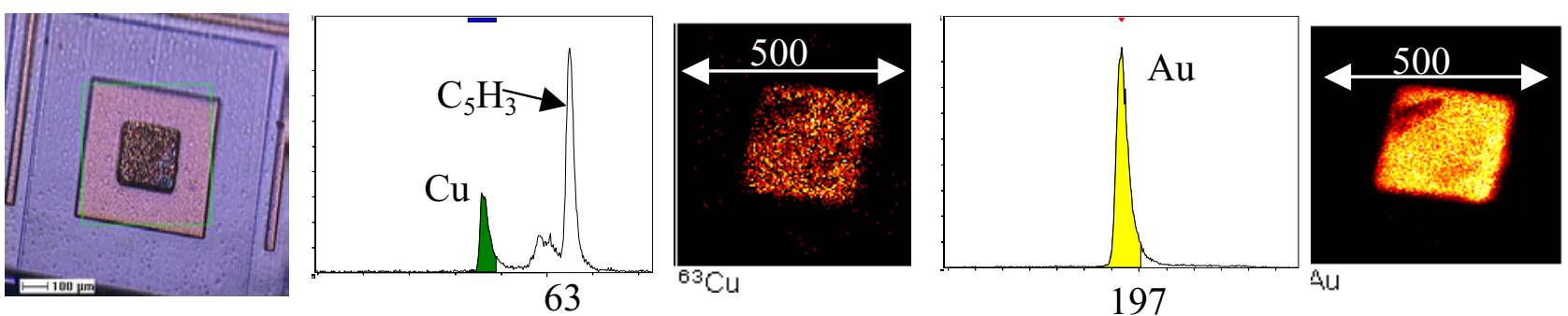

FIG. 1. Far left is optical photo of sample (the green box shows the region to be analyzed). Middle panel shows $\mathrm{Cu}$ region of the ToF-SIMS spectrum and an elemental image of $\mathrm{Cu}$, far right panel shows Au region of the spectrum and elemental image of $\mathrm{Au}$.
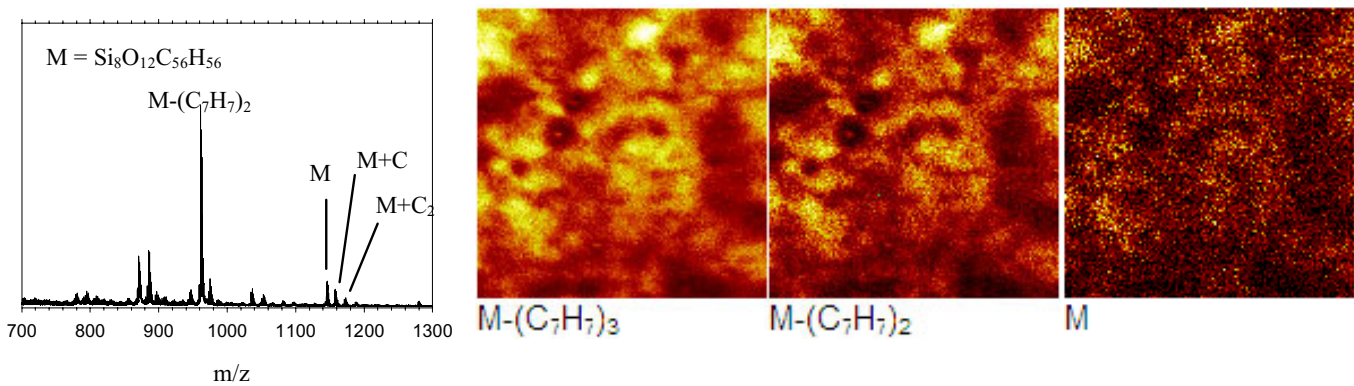

$\mathrm{m} / \mathrm{z}$

FIG. 2. ToF-SIMS spectrum (left), and images of BnPOSS in a polymer matrix. Note that we can detect and image the intact molecular species (as well as the dimer species at 2,290.4 amu.
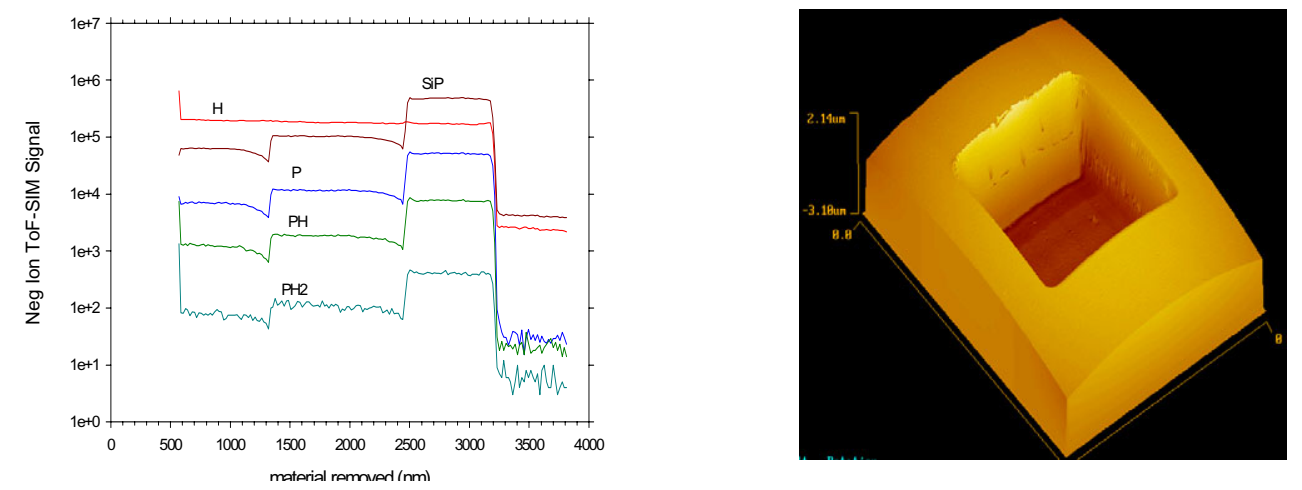

FIG 3. ToF-SIMS depth profile of $P$ in a multilayer Si film (left), and an optical profilometer image of the sputter crater (right). 